\title{
CRRTnet: a prospective, multi-national, observational study of continuous renal replacement therapy practices
}

\author{
Michael Heung ${ }^{1,7^{*}}$ (D, Sean M. Bagshaw2 ${ }^{2}$ Andrew A. House ${ }^{3}$, Luis A. Juncos ${ }^{4}$, Robin Piazza ${ }^{5}$ and Stuart L. Goldstein ${ }^{6}$
}

\begin{abstract}
Background: Continuous renal replacement therapy (CRRT) is the recommended modality of dialysis for critically ill patients with hemodynamic instability. Yet there remains significant variability in how CRRT is prescribed and delivered, and limited evidence-basis to guide practice.

Methods: This is a prospective, multi-center observational study of patients undergoing CRRT. Initial enrollment phase will occur at 4 academic medical centers in North America over 5 years, with a target enrollment of 2000 patients. All adult patients (18-89 years of age) receiving CRRT will be eligible for inclusion; patients who undergo CRRT for less than $24 \mathrm{~h}$ will be excluded from analysis. Data collection will include patient characteristics at baseline and at time of CRRT initiation; details of CRRT prescription and delivery, including machine-generated treatment data; and patient outcomes.

Discussion: The goal of this study is to establish a large comprehensive registry of critically ill adults receiving CRRT. Specific aims include describing variations in CRRT prescription and delivery across quality domains; validating quality measures for CRRT care by correlating processes and outcomes; and establishing a large registry for use in quality improvement and benchmarking efforts. For initial analyses, some particular areas of interest are anticoagulation protocols; approach to fluid overload; CRRT-related workload; and patient safety.
\end{abstract}

Trial registration: Registered on ClinicalTrials.gov 1/10/2014: NCT02034448.

Keywords: Acute kidney injury, Critical care, Continuous renal replacement therapy, Quality

\section{Background}

Acute kidney injury (AKI) occurs commonly in critically ill patients, complicating more than half of intensive care unit (ICU) admissions [1, 2]. About 5-6\% of ICU patients will receive renal replacement therapy (RRT), and hospital mortality rates in this population approach or exceed 50\% [3-6]. Despite the major clinical significance of this syndrome, there remains no proven therapy to reverse or attenuate established AKI, and care is primarily supportive, including RRT in severe cases.

For ICU patients, particularly those with hemodynamic instability, continuous renal replacement therapy (CRRT) has emerged as the dialysis modality of choice in high-

\footnotetext{
* Correspondence: mheung@umich.edu

'Division of Nephrology, Department of Medicine, University of Michigan, Ann Arbor, MI, USA

${ }^{7} 1500$ E. Medical Center Drive, SPC 5364, Ann Arbor, MI 48109-5364, USA Full list of author information is available at the end of the article
}

income nations. Since the original descriptions of continuous arteriovenous hemofiltration over 30 years ago, [7] CRRT has undergone significant evolution with several improvements allowing more widespread and routine use. Advancements include peristaltic pump driven venovenous circulation, sophisticated hardware and software engineering for precise application of the prescribed treatment and enhanced safety and alarms, improved biocompatibility and other membrane characteristics, regional anticoagulation protocols and enhancements in user interface. Indeed, for hemodynamically unstable patients the 2012 Kidney Disease: Improving Global Outcomes (KDIGO) AKI guidelines suggest using CRRT over intermittent dialysis options [8]. Compared to standard intermittent hemodialysis (IHD), CRRT offers advantages of improved hemodynamic stability, better overall solute clearance, and better fluid balance [8-10]. Observational 
studies also suggest higher rates of renal recovery among patients initially treated with CRRT compared to conventional IHD, although this benefit has not been observed in randomized trials [11].

While the clinical role of CRRT has become wellestablished, there exists tremendous variability in how CRRT is prescribed and delivered [12-14]. The KDIGO AKI guidelines provide some recommendations, stating that the target delivered CRRT effluent dose should be $20-25 \mathrm{~mL} / \mathrm{kg} / \mathrm{h}$, and also suggesting that regional citrate should be the first-line form of anticoagulation [8]. However, specific recommendations are lacking for a number of other important areas, such as CRRT modality, details of anticoagulation protocols, electrolyte supplementation practices, or approach to fluid balance. This reflects the lack of strong evidence upon which to base guidelines, as most studies examining CRRT practices have been relatively small and/or single-center. In addition, despite the high-risk nature of the patient population, very few studies have examined quality metrics and/or patient safety related to CRRT practices [15].

Here we describe the protocol for a prospective observational study focused on CRRT practices in critically ill adult patients across a network of medical centers in North America (CRRTnet). Our goal is to develop a large repository of data regarding CRRT practices and outcomes, allowing characterization of similarities and differences in standard CRRT practices across medical centers. The natural variability in practices between (and even within) centers will provide the ability to compare outcomes between different practice patterns and generate important preliminary data to inform future interventional trials. In addition, a long-term goal for CRRTnet is to establish benchmarks for best CRRT practice for centers both within and outside the study.

\section{Methods/design}

\section{Study oversight and consent}

This study was registered on ClinicalTrials.gov before initiation of recruitment (identifier NCT02034448). Each of the initial recruitment centers has received local institutional review board approval for CRRTnet with a waiver for informed consent. This waiver was granted due to the minimal risk nature of this observational study, as well as the recognition that this study would not be practical without the waiver. Specifically, since no interventions are involved, patient recruitment into the database may occur on a retrospective basis and it would be inefficient and possibly emotionally distressing to contact patients or their families for consent after the fact. Furthermore, it is important to include all CRRT patients in order to provide an unbiased perspective of practice patterns and maximize validity of our findings.
Ongoing study oversight will be performed by the CRRTnet registry board, which is chaired by SLG and comprised of the site principal investigators for each of the recruitment centers.

\section{Design and setting}

This is a prospective, multi-center observational study of critically ill adults undergoing CRRT. Initial recruitment is planned at 4 academic medical centers in North America with significant ( $>50$ patients per year) volume CRRT use: University of Michigan Health System (Ann Arbor, MI), University of Alberta Hospital, University of Alberta (Edmonton, Alberta), University Hospital London Health Sciences Centre (London, Ontario), and University of Mississippi Medical Center (Jackson, MS).

\section{Patient population}

All subjects in this study will be critically ill adult patients with AKI receiving CRRT as part of their standard of care. Inclusion criteria are age $\geq 18$ years and $\leq 89$ years. Exclusion criteria are patients who receive CRRT for less than $24 \mathrm{~h}$, and those who are initiated on CRRT at an outside hospital prior to transfer in whom incomplete data are available. For patients who require multiple courses of CRRT during an admission, only the data from the first course will be considered. Patients with pre-existing end-stage renal disease on maintenance dialysis are also excluded.

\section{Data collection}

Data will be collected encompassing a wide range of variables examining patient characteristics at enrollment, describing daily ICU course, capturing details of CRRT, and describing patient outcomes. Each participating medical center utilizes an electronic medical record which will serve as the primary source for data extraction.

The initial intake form includes demographic variables, and baseline patient characteristics at the time of CRRT initiation (Table 1). Fluid balance (in liters) between the time of ICU admission and CRRT initiation will be collected and used to calculate percent fluid overload using the following formula: [16, 17].

\%fluid overload $=[$ (total fluid intake $(\mathrm{L})-$ total fluid output (L))/ICU admission weight $(\mathrm{kg})] \times 100 \%$. Additional patient clinical data will be updated on a daily basis to reflect clinical management, severity of illness and laboratory findings (Table 2). All laboratory values will be recorded as available, and no laboratory testing will be requested as part of CRRTnet.

For up to the first 7 days of a patient's CRRT course, data regarding CRRT prescription and delivery will be recorded twice daily (Table 3). For days 8 thru 14, CRRT data will be captured once daily. Details of the CRRT prescription will be truncated at 14 days. Each of the 
Table 1 Planned data collection at time of enrollment (CRRT initiation)

\begin{tabular}{ll}
\hline Category & Data Elements \\
\hline Demographics & Date of birth/Age \\
& Gender \\
& Body mass index \\
& Hospital admission weight \\
& Components of Charlson Index \\
Comorbidities & ICU admission date \\
ICU Admission Data & Admission source (hospital ward, emergency \\
& department, outside hospital transfer) \\
& Primary condition \\
& Secondary condition \\
CRRT Initiation Data & Clinical indication for CRRT (all that apply) \\
& Time from ICU admission to CRRT initiation \\
& Cumulative fluid balance from ICU initiation \\
& to CRRT initiation \\
& Serum creatinine at ICU admission \\
& Serum creatinine at CRRT initiation \\
& APACHE II score at ICU admission
\end{tabular}

CRRT continuous renal replacement theray, ICU intensive care unit

initial recruitment centers performs CRRT utilizing the Prismaflex $^{\mathrm{ma}}$ device (Baxter International Inc.) which has a datacard to which machine treatment data are automatically saved. These data include detailed information such as pressure measurements, fluid removal, delivered CRRT effluent dose, and all alarms, interruptions and interventions. Machine treatment data files will be downloaded and analyzed for each patient. Each site will deidentify the patient file using the de-identified patient ID assigned by the registry database in place of the patient identifier. The de-identified machine data files will be

Table 2 Daily clinical data collection

\begin{tabular}{ll}
\hline Category & Data Elements \\
\hline Severity of Illness & SOFA score components \\
& Need for mechanical ventilation \\
& Vasopressor requirements (drug and rate) \\
Fluid Balance & Type and volume of fluid intake \\
& Type and volume of recorded outputs \\
Nutrition & Enteral nutrition formula and volume \\
& Parenteral nutrition protein, calories and volume \\
Laboratory Data & Complete blood count: white blood cell count, \\
& hemoglobin, hematocrit, platelets \\
& Metabolic panel: sodium, potassium, chloride, \\
& bicarbonate, blood urea nitrogen, creatinine, \\
& phosphate, magnesium, albumin \\
& Coagulation parameters: INR, PT, anti-Xa activity \\
& Blood gas: pH, PaO2, PaCO2, lactate, ionized calcium
\end{tabular}

Table 3 Daily CRRT data collection

\begin{tabular}{ll}
\hline Category & Data Elements \\
\hline Vascular Access & Dialysis catheter position \\
& Dialysis catheter type (acute or tunneled) \\
& Dialysis catheter manufacturer \\
& Dialysis catheter size \\
& Any need for catheter manipulation (e.g. \\
& repositioning, replacement) \\
& Any circuit interruptions/replacements \\
& and reasons \\
& Priming solution (new circuits) \\
CRRT Circuit & Anticoagulation type (heparin, \\
& citrate, none, other) \\
& Anticoagulation dose \\
CRRT-Related Anticoagulation & Anticoagulation metrics \\
& Type of solution \\
& Modality (dialysate, pre- and/or \\
CRRT Fluids & Solution additives \\
& Prescribed effluent dose \\
CRRT Dose & Delivered effluent dose \\
Machine Data Card & Type, number and duration of machine \\
alarms and interruptions & Filter life \\
\hline
\end{tabular}

CRRT continuous renal replacement theray

uploaded by the site into the CRRTnet Registry within each patient's record. Data is parsed by the application and stored in the database into select data fields based on dates and time points for each day the patient is on CRRT. If the data files cannot be accessed/used, the site can manually fill in the data fields for the specified time points with data collected by the medical staff.

Data entry will be performed by trained study staff at each site, and will be entered into an online system using a password-protected web-based research interface (Watermark EDGE, Watermark Research Partners, Inc.). Electronic data transmission will utilize secure socket layer (SSL) encryption technology to maintain compliance with privacy and data security. Data within the Watermark EDGE database is protected by Transparent Data Encryption (TDE) encrypted with the AES-265 Cipher. Limited Data Set (LDS) will be collected with regards to specific dates for the following: year of birth, admission dates, treatment dates, date of discharge or death. For patient data entry each patient will be de-identified via assigned unique codes and will not contain direct traceable identifiers. This registry complies with the following as set forth in 45 CFR Parts 160 and 164 ("HIPAA Privacy Rule"): "Covered Entity" (45 CFR $₫ 160.103)$, "De-identified information" (45 CFR $\mathbb{1}$ 164.514), "Protected Health Information ("PHI")" (45 CFR $\$$ 164.103) and "Limited 
Data Set ("LDS")" (45 CFR 164.514(e)(2)). All sites will utilize the same electronic report forms, with the exception of measurement units customized to match each center's customary laboratory reporting. For purposes of analysis all unit values entered by the center will also be converted and stored in a standardized unit for reporting in addition to the original entry.

\section{Interventions}

CRRTnet is strictly an observational study and no clinical interventions will be performed. In addition, no biosample collection or storage will occur as part of CRRTnet.

\section{Operational definitions}

1. Complete recovery: - Return of serum creatinine (SCr) to within $50 \%$ of baseline. Baseline SCr will be determined by the order of the following:

i. pre-hospital/outpatient SCr values

ii. lowest available in-hospital SCr (before or after) receiving CRRT

iii. back calculation for a SCr based on an eGFR $75 \mathrm{~mL} /$ min using the CKD-EPI equation

2. Partial recovery: - No longer receiving RRT but SCr has not returned to within $50 \%$ of baseline.

3. Non-recovery: - Remains dialysis dependent.

All serious adverse events related to CRRT will be recorded, with a specific focus on the following complications:

1. Major bleeding event related to heparin anticoagulation:

i. Drop in hemoglobin of $\geq 1.0 \mathrm{~g} / \mathrm{dL}$ within a $24 \mathrm{~h}$ period, and

ii. Transfusion requirement of $\geq 2$ units packed red blood cells

2. Development of heparin-induced thrombocytopenia:

i. Fall in platelets of at least $30 \%$ and

ii. Positive confirmatory test for heparin-induced thrombocytopenia (serotonin release assay)

3. Hypocalcemia event related to citrate anticoagulation:

i. Fall in systemic ionized calcium below $0.8 \mathrm{mmol} / \mathrm{L}$, and one of the following ii. Worsening hypotension/increasing pressor requirement, or

iii. Cardiac arrhythmia

All serious adverse events will be reviewed with each site's principal investigator to confirm appropriateness for inclusion.

\section{Primary and secondary outcomes}

The primary outcome will be patient survival at ICU discharge. Patient outcomes will also be recorded at hospital discharge. Additional outcomes include ICU and hospital length of stay, renal function recovery during hospitalization (defined above), and adverse events related to CRRT (defined above).

\section{Sample size}

Due to the observational nature of CRRTnet, a formal sample size calculation was not performed. However, our target recruitment is 2000 patients, which would result in the largest and most detailed collection of CRRT patients to date.

\section{Statistical analysis}

All descriptive data will be analyzed using standard statistical methods. CRRTnet will assess for potential associations between patient characteristics, CRRT initiation and prescription parameters, and patient outcomes (mortality, length of stay, duration of AKI) using ANOVA (for normally distributed variables) or Kruskal-Wallis (for non-normally distributed variables). Multivariate proportional hazards models will be constructed, with initial inclusion of variables with $<0.2$ significance in univariate analyses. Additional analyses of registry data will be developed based on specific research questions.

Data analyses will be performed by Technomics Research, LLC (Minneapolis, MN) and guided by the CRRTnet registry board.

\section{Discussion}

Severe AKI remains a common complication of critically ill patients, and recent studies suggest that the incidence of dialysis-requiring AKI is increasing [18]. CRRT has emerged as the recommended dialysis modality for hemodynamically unstable patients in the ICU, and a recent study demonstrates that CRRT is the most commonly used dialysis modality in critically ill patients with AKI [19]. CRRT provides the advantages of improved solute and fluid balance control compared to patients on intermittent therapies. Yet in reality "CRRT" is an umbrella term that encompasses multiple different modalities and approaches. A high degree of variation exists in how CRRT is prescribed and delivered between (and within) medical centers, and there is currently a paucity 
of high-quality evidence to guide best practices. CRRTnet was developed with the goal of establishing the largest registry of CRRT patients to date, allowing for comparison of outcomes between a variety of different CRRT practices.

Ideally, each aspect of therapy would be assessed in randomized clinical trials. However, given the complexities of critically ill patients and potential difficulties with recruitment in this population, such an approach may not be feasible to assess every aspect of CRRT care. As such, observational studies remain an important tool, particularly when significant variations in practice naturally exist. As an example, CRRTnet was modeled after the Prospective Pediatric CRRT registry (ppCRRT) [20]. Over a 4 year period and including 13 centers in the United States, the ppCRRT enrolled 370 critically ill children who underwent CRRT and characterized outcomes in this previously under-described population [21]. Subsequent studies from ppCRRT explored patient outcomes related to vascular access, [22] anticoagulation, [23] and nutritional practices [24]. These studies provided important insight into CRRT practices and outcomes in children, spurred additional research, and continue to guide best practices in this population by informing the KDIGO AKI guidelines. Although there is significantly more literature regarding CRRT practices in adults, most of this literature is comprised of relatively small studies, primarily from single-centers. As such, there remains a significant need for large, multi-center studies such as CRRTnet.

Beyond descriptive analyses of patient characteristics and overall outcomes, we are planning on several comparative analyses. One particular area of interest is in the management of fluid overload. Our data will allow determination of fluid overload status at time of CRRT initiation, as well as daily fluid balances thereafter. While the dangers of established fluid overload have been increasing recognized in recent years, $[10,25,26]$ there is a paucity of data examining different approaches to fluid management utilizing renal replacement therapy. We will examine rates of fluid removal (e.g. in $\mathrm{ml} / \mathrm{kg} / \mathrm{h}$ ) and fluid overload correction to determine potential impact on patient outcomes such as renal function recovery and mortality. We hypothesize that, after adjusting for severity of illness, higher fluid removal rates will be associated with lower likelihood of renal recovery.

Another area of particular interest is anticoagulation to maintain CRRT filter patency. The KDIGO guidelines suggest regional citrate anticoagulation as the first-line method in patients without a contraindication. We anticipate that citrate anticoagulation will be associated with longer circuit life compared to heparin or no anticoagulation, a finding that has been observed in a number of clinical trials [27-30]. However, there is no consensus approach to citrate anticoagulation; while several different protocols have been published, there have been no comparative trials. Because of existing differences in citrate anticoagulation protocols among CRRTnet centers, we will be able to compare outcomes between different protocols.

One novel aspect of CRRTnet is the capture and analysis of machine data. These data record all machine measurements to the second, and include pressure measurements (e.g. access and return line pressures, filter pressures, transmembrane pressures), alarms and response to alarms, any changes in prescription (including blood, dialysate and replacement flow rates), interruptions, and actual delivered therapy. To our knowledge, this is a relatively untapped source of information, and one goal of CRRTnet will be to develop algorithms for processing the information downloaded from the CRRT machine memory cards. One of the challenges of CRRT is the complexity of therapy and the increased associated workload compared to intermittent therapies [31]. By utilizing the detailed treatment data, we will be able to characterize alarm frequency and provide more precise estimates on interface time required to maintain this therapy. This information may be helpful to administrators in determining nursing models and CRRT educational programs. In addition, nursing workload can be considered an important secondary outcome that may vary between and within centers depending on variations in CRRT protocol. For example, we hypothesize that regional citrate anticoagulation will be associated with less workload compared to non-citrate anticoagulation. It will also be interesting to compare associated workload between different citrate protocols, especially if circuit filter life is similar.

An important strength of our study is that we are examining everyday practices. While findings from controlled clinical trials can sometimes be difficult to duplicate in the "real world" setting, we will provide a relatively unfiltered look. This may be particularly important when examining adverse events and complications of therapy. Conversely, it is important to recognize that CRRT is performed at a relatively high frequency at each of the study sites, which facilitates development of local expertise and maintenance of competency. Whether or not similar practices will result in similar outcomes at smaller centers with lower volumes is uncertain. Nonetheless, we believe that CRRTnet will provide important outcomes benchmarks for centers performing CRRT.

Another important strength of CRRTnet is the ability, through waiver of consent, to include all eligible participants. In terms of the practicality of obtaining informed consent for an observational study in a population similar to our proposal, much can be learned from the unfortunate experience of the PICARD study investigators where 
patients and substitute decision makers were approached for informed consent [32]. Enrollment was poor, with only $52 \%$ of eligible patients included, greatly hindering the generalizability of the results. Patient refusal was infrequent at all sites, suggesting that patients themselves did not have concerns or objections to having their data collected when they were able to be approached directly. However, refusal by family occurred in almost one fifth of instances, and the absence of family or appropriate proxy was listed as a cause for refusing enrollment in as high as $40 \%$ at one site. Finally, many patients could not be enrolled because of death or discharge before being seen by study personnel. Thus, countless resources were spent on conducting an observational trial that missed almost half the eligible subjects, whose data very likely would have influenced the results of the study were they included. The CRRTnet database will be highly generalizable based on the inclusion of most eligible patients.

Some limitations of our study design are worth noting. First, we will truncate CRRT data at 14 days, which will limit applicability in patients on prolonged courses of CRRT. This decision was made primarily as a feasibility measure to limit the workload associated with detailed clinical data collection; importantly, preliminary data from participating centers showed that median duration of CRRT was in the range of 6-8 days, so we will still capture the full CRRT course for the majority of patients enrolled. Second, we will not be enrolling patients with AKI who only receive intermittent dialysis therapies and we recognize that there can be significant variation in which patients are chosen to undergo CRRT versus intermittent therapies. However, it is important to note that our goal is not to establish the role of CRRT in AKI management, but rather to focus on practice variation within CRRT prescription and delivery. So while we will not be able to advocate for which patients should be managed with CRRT, we hope to inform clinicians of best practices once a decision to initiate CRRT is made.

Over the past 30 years, CRRT has developed into an important tool for the management of critically ill patients with renal failure. Despite overall improvements, there remains marked variation in CRRT practices which is driven in part by lack of evidence-based guidance. The primary goal of CRRTnet is to develop a large data repository of CRRT patients with detailed information regarding specific CRRT practices which can then be compared in relation to outcomes. We anticipate that CRRTnet will provide the nephrology and critical care communities with an important resource for planning future clinical trials as well as for benchmarking clinical outcomes.

\section{Trial status}

Recruitment is currently active at the 4 medical centers mentioned above. A fifth center is undergoing evaluation to join CRRTnet. The initial planned recruitment phase is 5 years with a target enrollment of 2000 patients.

\section{Abbreviations \\ AKI: Acute kidney injury; CRRT: Continuous renal replacement therapy; ICU: intensive care unit; IHD: Intermittent hemodialysis; KDIGO: Kidney Disease: Improving Global Outcomes; SCr: Serum creatinine}

\section{Acknowledgements \\ SMB is supported by a Canada Research Chair in Critical Care Nephrology. \\ Funding \\ Funding for the study is provided by the Acute Kidney Injury Critical Care Research Foundation (AKI-CCRF, Indianapolis, Indiana, SLG President). The AKI-CCRF initially received an unrestricted grant from Gambro Renal Products, Inc. (Lakewood, CO) to establish CRRTnet, which ended in 2014. Since July 2015, the AKI-CCRF has received funding from Cincinnati Children's Hospital Medical Center (CCHMC) to operate CRRTnet. CCHMC receives funding from Baxter Healthcare Inc., (Deerfield, IL) through an Investigator Initiated Research grant (PI: SLG) for the expressed purpose of funding the AKI-CCRF's CRRTnet activity. Neither Baxter nor its personnel had any involvement in the design of CRRTnet or the collection, analysis, and interpretation of data; writing the report; and the decision to submit the report for publication.}

\section{Availability of data and materials}

The datasets generated during and/or analyzed during the current study are not publicly available due to privacy concerns given the prospective nature of the data collection, but are available from the corresponding author on reasonable request.

\section{Authors' contributions}

$\mathrm{MH}, \mathrm{SMB}, \mathrm{AAH}$ and LAJ serve as individual site principal investigators for CRRTnet, while SG serves as global consultant. RP was the primary architect of the online data collection tool and serves as the primary data analyst. All the authors participated in all phases of this study, including development of the study design and methodology, overseeing data collection, guiding data analyses, data interpretation, and manuscript preparation and review. All authors read and approved the final manuscript.

\section{Ethics approval and consent to participate}

The study was reviewed and approved by the local institutional review board (IRB) for each participating site. In all cases, a waiver for informed consent was granted due to the observational nature of the study. The names of each IRB and specific reference number for CRRTnet are as follows: - University of Michigan IRB-Med (HUM00074701)

- University of Alberta Health Research Ethics Board (File \# Pro00038405)

- Western University Research Ethics Board (File \# 103957)

- University of Mississippi Medical Center IRB (\#2013-0190)

- Cincinnati Children's Hospital Medical Center (\#2014-5675)

Consent for publication

Not applicable.

\section{Competing interests}

$\mathrm{SMB}$ and $\mathrm{AAH}$ have consulted for and received speaking fees from Baxter Healthcare Corp.

All other authors declare that they have no competing interests.

\section{Publisher's Note}

Springer Nature remains neutral with regard to jurisdictional claims in published maps and institutional affiliations.

\section{Author details \\ ${ }^{1}$ Division of Nephrology, Department of Medicine, University of Michigan, Ann Arbor, MI, USA. ${ }^{2}$ Department of Critical Care Medicine, Faculty of Medicine and Dentistry, University of Alberta, Edmonton, AB, Canada. ${ }^{3}$ Division of Nephrology, Department of Medicine, University Hospital London Health Sciences Centre, London, ONT, Canada. ${ }^{4}$ Department of Medicine/Nephrology, and Department of Physiology and Biophysics,}


University of Mississippi Medical Center, Jackson, MS, USA. ${ }^{5}$ Watermark Research Partners, Inc., Indianapolis, IN, USA. ${ }^{\circ}$ Center for Acute Care Nephrology, Cincinnati Children's Hospital Medical Center, Cincinnati, $\mathrm{OH}$, USA. ${ }^{7} 1500$ E. Medical Center Drive, SPC 5364, Ann Arbor, MI 48109-5364, USA.

Received: 25 November 2016 Accepted: 29 June 2017

Published online: 06 July 2017

\section{References}

1. Hoste EA, Clermont G, Kersten A, Venkataraman R, Angus DC, De Bacquer D, Kellum JA. RIFLE criteria for acute kidney injury are associated with hospital mortality in critically ill patients: a cohort analysis. Crit Care. 2006;10(3):R73.

2. Herrera-Gutierrez ME, Seller-Perez G, Sanchez-Izquierdo-Riera JA, MaynarMoliner J. Prevalence of acute kidney injury in intensive care units: the "COrte de prevalencia de disFuncion RenAl y DEpuracion en criticos" pointprevalence multicenter study. J Crit Care. 2013;28(5):687-94.

3. Hoste EA, Schurgers M. Epidemiology of acute kidney injury: how big is the problem? Crit Care Med. 2008;36(4 Suppl):S146-51.

4. Uchino S, Kellum JA, Bellomo R, Doig GS, Morimatsu H, Morgera S, Schetz M, Tan I, Bouman C, Macedo E, et al. Acute renal failure in critically ill patients: a multinational, multicenter study. JAMA. 2005;294(7):813-8.

5. Susantitaphong P, Cruz DN, Cerda J, Abulfaraj M, Alqahtani F, Koulouridis I Jaber BL. World incidence of AKl: a meta-analysis. Clin J Am Soc Nephrol. 2013;8(9):1482-93.

6. Bagshaw SM, Laupland KB, Doig CJ, Mortis G, Fick GH, Mucenski M, Godinez-Luna T, Svenson LW, Rosenal T. Prognosis for long-term survival and renal recovery in critically ill patients with severe acute renal failure: a population-based study. Crit Care. 2005;9(6):R700-9.

7. Kramer P, Schrader J, Bohnsack W, Grieben G, Grone HJ, Scheler F. Continuous arteriovenous haemofiltration. A new kidney replacement therapy. Proc Eur Dial Transplant Assoc. 1981;18:743-9.

8. Kellum JALN, Aspelin P, et al. KDIGO clinical practice guideline for acute kidney injury. Kidney Int. 2012;2:1-138.

9. Rabindranath K, Adams J, Macleod AM, Muirhead N. Intermittent versus continuous renal replacement therapy for acute renal failure in adults. Cochrane Database Syst Rev. 2007;3:CD003773.

10. Bouchard J, Soroko SB, Chertow GM, Himmelfarb J, Ikizler TA, Paganini EP, Mehta RL. Program to improve Care in Acute Renal Disease Study G: fluid accumulation, survival and recovery of kidney function in critically ill patients with acute kidney injury. Kidney Int. 2009;76(4):422-7.

11. Schneider AG, Bellomo R, Bagshaw SM, Glassford NJ, Lo S, Jun M, Cass A, Gallagher M. Choice of renal replacement therapy modality and dialysis dependence after acute kidney injury: a systematic review and metaanalysis. Intensive Care Med. 2013;39(6):987-97

12. Uchino S, Bellomo R, Morimatsu H, Morgera S, Schetz M, Tan I, Bouman C, Macedo E, Gibney N, Tolwani A, et al. Continuous renal replacement therapy: a worldwide practice survey. The beginning and ending supportive therapy for the kidney (B.E.S.T. Kidney) investigators. Intensive Care Med. 2007;33(9):1563-70.

13. Fealy $N$, Aitken $L$, Toit E, Baldwin I. Continuous renal replacement therapy: current practice in Australian and New Zealand intensive care units. Crit Care Resusc. 2015;17(2):83-91.

14. Legrand M, Darmon M, Joannidis M, Payen D. Management of renal replacement therapy in ICU patients: an international survey. Intensive Care Med. 2013;39(1):101-8.

15. Rewa O, Mottes T, Bagshaw SM. Quality measures for acute kidney injury and continuous renal replacement therapy. Curr Opin Crit Care. 2015:21(6):490-9.

16. Goldstein SL, Currier H, Graf C, Cosio CC, Brewer ED, Sachdeva R. Outcome in children receiving continuous venovenous hemofiltration. Pediatrics. 2001;107(6):1309-12

17. McGuire MD, Heung M. Fluid as a drug: balancing resuscitation and fluid overload in the intensive care setting. Adv Chronic Kidney Dis. 2016;23(3):152-9.

18. Hsu RK, McCulloch CE, Dudley RA, Lo LJ, Hsu CY. Temporal changes in incidence of dialysis-requiring AKI. J Am Soc Nephrol. 2013;24(1):37-42.

19. Hoste EA, Bagshaw SM, Bellomo R, Cely CM, Colman R, Cruz DN, Edipidis K, Forni LG, Gomersall CD, Govil D, et al. Epidemiology of acute kidney injury in critically ill patients: the multinational AKI-EPI study. Intensive Care Med. 2015;41(8):1411-23.
20. Goldstein SL, Somers MJ, Brophy PD, Bunchman TE, Baum M, Blowey D, Mahan JD, Flores FX, Fortenberry JD, Chua A, et al. The prospective pediatric continuous renal replacement therapy (ppCRRT) registry: design, development and data assessed. Int J Artif Organs. 2004;27(1):9-14.

21. Symons JM, Chua AN, Somers MJ, Baum MA, Bunchman TE, Benfield MR, Brophy PD, Blowey D, Fortenberry JD, Chand D, et al. Demographic characteristics of pediatric continuous renal replacement therapy: a report of the prospective pediatric continuous renal replacement therapy registry. Clin J Am Soc Nephrol. 2007;2(4):732-8.

22. Hackbarth R, Bunchman TE, Chua AN, Somers MJ, Baum M, Symons JM, Brophy PD, Blowey D, Fortenberry JD, Chand D, et al. The effect of vascular access location and size on circuit survival in pediatric continuous renal replacement therapy: a report from the PPCRRT registry. Int J Artif Organs. 2007;30(12):1116-21.

23. Brophy PD, Somers MJ, Baum MA, Symons JM, McAfee N, Fortenberry JD, Rogers K, Barnett J, Blowey D, Baker C, et al. Multi-centre evaluation of anticoagulation in patients receiving continuous renal replacement therapy (CRRT). Nephrol Dial Transplant. 2005;20(7):1416-21.

24. Zappitelli M, Goldstein SL, Symons JM, Somers MJ, Baum MA, Brophy PD, Blowey D, Fortenberry JD, Chua AN, Flores FX, et al. Protein and calorie prescription for children and young adults receiving continuous renal replacement therapy: a report from the prospective pediatric continuous renal replacement therapy registry group. Crit Care Med. 2008:36(12):3239-45.

25. Heung M, Wolfgram DF, Kommareddi M, Hu Y, Song PX, Ojo AO. Fluid overload at initiation of renal replacement therapy is associated with lack of renal recovery in patients with acute kidney injury. Nephrol Dial Transplant. 2012;27(3):956-61.

26. Garzotto F, Ostermann M, Martin-Langerwerf D, Sanchez-Sanchez M, Teng J, Robert R, Marinho A, Herrera-Gutierrez ME, Mao HJ, Benavente D, et al. The dose response multicentre investigation on fluid assessment (DoReMIFA) in critically ill patients. Crit Care. 2016;20(1):196.

27. Kutsogiannis DJ, Gibney RT, Stollery D, Gao J. Regional citrate versus systemic heparin anticoagulation for continuous renal replacement in critically ill patients. Kidney Int. 2005:67(6):2361-7.

28. Monchi M, Berghmans D, Ledoux D, Canivet $J$, Dubois B, Damas P. Citrate vs. heparin for anticoagulation in continuous venovenous hemofiltration: a prospective randomized study. Intensive Care Med. 2004;30(2):260-5.

29. Schilder L, Nurmohamed SA, Bosch FH, Purmer IM, den Boer SS, Kleppe CG, Vervloet MG, Beishuizen A, Girbes AR, Ter Wee PM, et al. Citrate anticoagulation versus systemic heparinisation in continuous venovenous hemofiltration in critically ill patients with acute kidney injury: a multi-center randomized clinical trial. Crit Care. 2014;18(4):472.

30. Gattas DJ, Rajbhandari D, Bradford C, Buhr H, Lo S, Bellomo R. A randomized controlled trial of regional citrate versus regional heparin anticoagulation for continuous renal replacement therapy in critically ill adults. Crit Care Med. 2015;43(8):1622-9.

31. De Becker W. Starting up a continuous renal replacement therapy program on ICU. Contrib Nephrol. 2007;156:185-90.

32. Chertow GM, Pascual MT, Soroko S, Savage BR, Himmelfarb J, Ikizler TA, Paganini EP, Mehta RL. Picard: reasons for non-enrollment in a cohort study of ARF: the program to improve Care in Acute Renal Disease (PICARD) experience and implications for a clinical trials network. Am J Kidney Dis. 2003:42(3):507-12

\section{Submit your next manuscript to BioMed Central and we will help you at every step:}

- We accept pre-submission inquiries

- Our selector tool helps you to find the most relevant journal

- We provide round the clock customer support

- Convenient online submission

- Thorough peer review

- Inclusion in PubMed and all major indexing services

- Maximum visibility for your research

Submit your manuscript at www.biomedcentral.com/submit 